\title{
SPATIALLY RESOLVED SPECTROSCOPY OF EUROPA: THE DISTINCT SPECTRUM OF LARGE-SCALE CHAOS
}

\author{
P. D. FisCher ${ }^{1}$, M. E. BRown ${ }^{1}$, AND K. P. HAND ${ }^{2}$ \\ ${ }^{1}$ Division of Geological and Planetary Sciences, California Institute of Technology, Pasadena, CA 91125, USA; pfischer@caltech.edu \\ 2 Jet Propulsion Laboratory, California Institute of Technology, Pasadena, CA 91109, USA \\ Received 2015 July 23; accepted 2015 October 4; published 2015 November 2
}

\begin{abstract}
We present a comprehensive analysis of spatially resolved moderate spectral resolution near-infrared spectra obtained with the adaptive optics system at the Keck Observatory. We identify three compositionally distinct end member regions: the trailing hemisphere bullseye, the leading hemisphere upper latitudes, and a third component associated with leading hemisphere chaos units. We interpret the composition of the three end member regions to be dominated by irradiation products, water ice, and evaporite deposits or salt brines, respectively. The third component is associated with geological features and distinct from the geography of irradiation, suggesting an endogenous identity. Identifying the endogenous composition is of particular interest for revealing the subsurface composition. However, its spectrum is not consistent with linear mixtures of the salt minerals previously considered relevant to Europa. The spectrum of this component is distinguished by distorted hydration features rather than distinct spectral features, indicating hydrated minerals but making unique identification difficult. In particular, it lacks features common to hydrated sulfate minerals, challenging the traditional view of an endogenous salty component dominated by $\mathrm{Mg}$-sulfates. Chloride evaporite deposits are one possible alternative.
\end{abstract}

Key words: planets and satellites: composition - planets and satellites: individual (Europa) - planets and satellites: surfaces

\section{INTRODUCTION}

Europa's icy, geologically active surface conceals a global, subsurface ocean (Kivelson et al. 2000) in contact with a rocky sea floor and potentially hospitable to chemosynthetic life. The greatest challenge for generating and sustaining such a biosphere may be the compositional environment and chemical budget of the subsurface ocean (Hand et al. 2009). Therefore, we must understand the environment of the subsurface ocean in order to understand its potential for habitability. Surface composition is currently the best measurable link to the subsurface.

Europa's surface composition is dominated by water ice (Kuiper 1957), but contains a variety of non-water-ice species delivered by impacts, emplaced endogenously by geophysical processes, and produced exogenously by irradiation of the preexisting surface (Carlson et al. 2009 and references therein). Many of these species are known or speculated to be present. Material ejected from Io is ionized and swept up by the Jovian magnetosphere and implanted onto Europa's surface, particularly ionized sulfur and oxygen. Energetic ions and electrons are also showered onto the surface, as well as cosmic rays and solar radiation, which break apart existing molecular bonds and form new radiolytic species, such as $\mathrm{SO}_{2}$ (Lane et al. 1981), $\mathrm{O}_{2}$ (Lanzerotti et al. 1978; Johnson et al. 1982), $\mathrm{H}_{2} \mathrm{O}_{2}$, (Carlson et al. 1999a), and $\mathrm{H}_{2} \mathrm{SO}_{4}$ and elemental sulfur $\mathrm{S}_{x}$ (Carlson et al. 1999b). Endogenous material is revealed by association with geologic features; irradiated $\mathrm{NaCl}$ (Hand \& Carlson 2015) or sulfur polymers (Carlson et al. 1999b) may cause the redbrown color associated with linea. $\mathrm{Na}$ and $\mathrm{K}$ have been detected in the sputtered atmosphere, and their abundance ratio may indicate an endogenous source, at least for $\mathrm{Na}$ (Brown 2001). Epsomite has been detected (Brown \& Hand 2013), and other sulfate salts are speculated to be present as well (McCord et al. 1998), though an exogenous versus endogenous origin is uncertain (Brown \& Hand 2013; Hand \& Carlson 2015).
Surface composition is one boundary in a continuous cycle of exchange with the subsurface. Upward transport through the ice shell by processes such as compositional diapirism (Pappalardo \& Barr 2004) or convective ice plumes (Peddinti \& McNamara 2015) may leave a compositional fingerprint on the surface from the ocean. Downward transport of radiolytic species from the surface, aided by tectonic subsumption (Kattenhorn \& Prockter 2014), may provide the critical fuel for a redox-powered ecosystem (Chyba 2000; Hand et al. 2009) if there is interaction with the ocean in appropriate time scales. Identifying the non-water-ice components is key to understanding the subsurface environment.

Previous efforts to identify the non-ice surface components and determine their geographical associations is mostly based on measurements from the Galileo Near Infrared Mapping Spectrometer (NIMS). McCord et al. (1998) recognized the presence of hydrated impurities, and suggested hydrated salts and evaporites to be the non-ice component across the trailing hemisphere and in the linea. Carlson et al. (1999b) proposed hydrated sulfuric acid as the dominant non-ice component, and suggested that exogenous plasma implantation and endogenous geologic emplacement were both possible sources of sulfur. Dalton et al. (2005) suggested that the reddish and dark terrains were a complex mixture of sulfate hydrates and other species. Dalton (2007) recognized that the NIMS spectra are better described by mixtures of salt and sulfuric acid hydrates, suggesting both to be present. McCord et al. (2010) used a supervised spectral mixture analysis to claim five end members in one NIMS high resolution scene, and noted a distinction between the trailing hemisphere dark material and red material in linea. Shirley et al. (2010) explored the association between NIMS spectra and terrain type in a NIMS high resolution scene, claiming a spatial distinction between sulfuric acid hydrate and salt hydrates, with a greater abundance of hydrated salts and not hydrated sulfuric acid in the low albedo plains. Dalton et al. 
Table 1

Summary of Europa Observations

\begin{tabular}{|c|c|c|c|c|c|c|}
\hline Date (UT) & Time (UT) & Hemisphere & Limb & Band & Sub-Earth Lon. & Sub-Earth Lat. \\
\hline 2011 Sep 18 & 09:10 & Leading & west & $\mathrm{K}$ & $41 \mathrm{~W}$ & $3 \mathrm{~N}$ \\
\hline 2011 Sep 18 & $09: 26$ & Leading & east & $\mathrm{K}$ & $43 \mathrm{~W}$ & $3 \mathrm{~N}$ \\
\hline 2011 Sep 18 & $10: 22$ & Leading & west & $\mathrm{H}$ & $47 \mathrm{~W}$ & $3 N$ \\
\hline 2011 Sep 18 & $10: 31$ & Leading & east & $\mathrm{H}$ & $48 \mathrm{~W}$ & $3 \mathrm{~N}$ \\
\hline 2011 Sep 19 & 09:05 & anti-Jovian & west & $\mathrm{K}$ & $143 \mathrm{~W}$ & $3 \mathrm{~N}$ \\
\hline 2011 Sep 19 & $09: 37$ & anti-Jovian & east & $\mathrm{K}$ & $145 \mathrm{~W}$ & $3 \mathrm{~N}$ \\
\hline 2011 Sep 19 & $10: 48$ & anti-Jovian & west & $\mathrm{H}$ & $150 \mathrm{~W}$ & $3 \mathrm{~N}$ \\
\hline 2011 Sep 19 & 11:06 & anti-Jovian & east & $\mathrm{H}$ & $151 \mathrm{~W}$ & $3 \mathrm{~N}$ \\
\hline 2011 Sep 19 & 14:04 & anti-Jovian & west & $\mathrm{K}$ & $164 \mathrm{~W}$ & $3 \mathrm{~N}$ \\
\hline 2011 Sep 19 & $14: 37$ & anti-Jovian & east & $\mathrm{K}$ & $166 \mathrm{~W}$ & $3 \mathrm{~N}$ \\
\hline 2011 Sep 19 & $15: 05$ & anti-Jovian & west & $\mathrm{H}$ & $167 \mathrm{~W}$ & $3 \mathrm{~N}$ \\
\hline 2011 Sep 19 & $15: 22$ & anti-Jovian & east & $\mathrm{H}$ & $169 \mathrm{~W}$ & $3 \mathrm{~N}$ \\
\hline 2011 Sep 20 & 09:09 & Trailing & west & $\mathrm{K}$ & $244 \mathrm{~W}$ & $3 \mathrm{~N}$ \\
\hline 2011 Sep 20 & $09: 43$ & Trailing & east & $\mathrm{K}$ & $247 \mathrm{~W}$ & $3 N$ \\
\hline 2011 Sep 20 & $10: 33$ & Trailing & west & $\mathrm{H}$ & $250 \mathrm{~W}$ & $3 \mathrm{~N}$ \\
\hline 2011 Sep 20 & $10: 51$ & Trailing & east & $\mathrm{H}$ & $252 \mathrm{~W}$ & $3 N$ \\
\hline
\end{tabular}

(2012) examined four NIMS high resolution slices and suggested three dominant spectroscopic components: water ice, hydrated sulfuric acid, and hydrated salts. Beyond NIMS, Brown \& Hand (2013) used hyperspectral observations with Keck II adaptive optics to identify a new spectral feature at $2.07 \mu \mathrm{m}$, and find that it is associated with the trailing hemisphere and exogenous bombardment.

Here we analyze the spatially resolved spectra from Brown \& Hand (2013), with higher spectral resolution and greater geographic coverage than NIMS, and find evidence for three distinct compositional units at the $\sim 100 \mathrm{~km}$ scale. The endogenous and exogenous units are revealed by their geographical distributions; the exogenous component dominates the bullseye centered on the trailing hemisphere, and the endogenous component dominates leading hemisphere chaos units. A distinct composition associated with surface geology is not consistent with an ice shell passively altered by exogenous processes, but is consistent with active cycling of the ice shell. We compare the characteristic endogenous spectrum to laboratory spectra of plausible endogenous minerals, and find that it is not consistent with the hydrated sulfate salts previously favored. A definitive identification remains elusive, but chloride evaporite deposits are one possible alternative.

\section{OBSERVATIONS AND DATA REDUCTION}

Europa was observed over three nights in 2011 September 18-20 with the OSIRIS integral field spectrograph and adaptive optics system on the Keck II telescope (Larkin et al. 2003). Observations were acquired at four orbital orientations within one Europa orbit, and give nearly complete latitude/longitude coverage. Spectra were obtained at moderate resolution $(R \approx 3800)$ in the infrared $\mathrm{H}$ and $\mathrm{K}$ bands $(1.473-1.803$ and $1.956-2.381 \mu \mathrm{m})$, at the $0 . " 035$ pixel $^{-1}$ setting, and an angular resolution coarser than the detector resolution by a factor of $\sim 2$. The disk of Europa was $\sim 1$ ". 03 on the sky, yielding an average surface resolution of $\sim 150 \mathrm{~km}$ per spatial element. The OSIRIS grid has a field of view 0 ". $56 \times 2$ ". 24 , so that the entire disk of Europa was captured with two exposures per spectral band. See Table 1 for the relevant orientations observed. For details regarding calibration, initial data reduction, and a complete journal of observations, see Brown \& Hand (2013).

In addition to the calibration from Brown \& Hand (2013), we make an extra correction to most accurately match spectra to specific locations on the surface. This task is slightly complicated by each hyperspectral full-disk consisting of four separate exposures, an east and west limb in the $\mathrm{H}$ and $\mathrm{K}$ bands (Table 1). Due to Europa's rotation, exposures in $\mathrm{H}$ and $\mathrm{K}$ separated by $\sim 1 \mathrm{hr}$ are offset in longitude by $\sim 6^{\circ}$ or $\sim 1.6$ OSIRIS pixels at the sub-observer point. To partially account for this, we use a lambertian surface to normalize each $\mathrm{H}$ and $\mathrm{K}$ observation to normal incidence. We then generate an artificial grid with the same resolution as OSIRIS, centered at the average sub-observer longitude of the four exposures. The full $\mathrm{H}$ and $\mathrm{K}$ spectrum of each location in the new grid is obtained by nearest interpolation of the original exposures. This results in a spatial error between $\mathrm{H}$ and $\mathrm{K}$ spectra of $<1$ OSIRIS pixel or $\lesssim 3^{\circ} .8$ longitude at the sub-observer point.

\section{END MEMBER EXTRACTION}

In order to coherently map the surface composition of Europa, we first seek the smallest number of unique observed surface spectra which, when linearly combined, can be used to optimally model all of the spectra on the surface of Europa. While past efforts at such modeling have relied on matching laboratory-derived spectral libraries (McCord et al. 1999; Carlson et al. 2005; Shirley et al. 2010; Dalton et al. 2012), or supervised selection of surface spectra (McCord et al. 2010), we instead take a purely empirical approach in order to remove preconceived assumptions on potential surface compositions.

Mathematically, we define the $N$ end members as the spectra at $N$ different locations which, when used as the basis set in a linear least-squares fit of the separate spectrum at each spatial location, minimizes the sum of the square of the residuals across the entire surface of Europa. With this definition, our task is then to determine $N$, the appropriate number of end members, and what each end member spectrum is. There is an extensive literature on the subject of end member extraction and many possible approaches, and no single approach is preferred for all situations. We chose to develop our own 


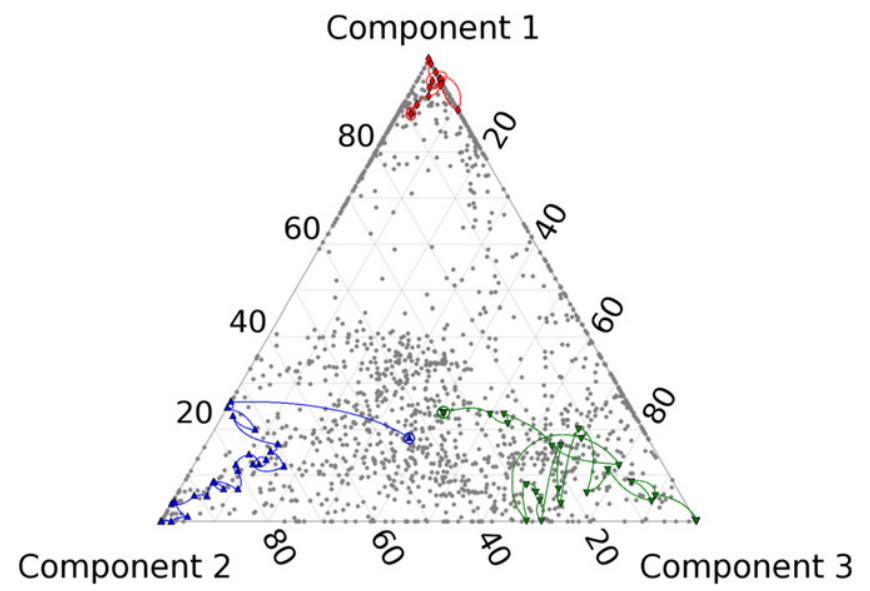

Figure 1. Progression of the end member extraction routine for three end members. End members converge to the corners of the ternary plot (color symbols). Initial guesses are obtained from a standard $k$-means algorithm and SAM distance metric. Candidate end members are iteratively substituted with new candidates, and replaced if the new candidate lowers the total rms. All observed spectra (gray symbols) are a combination of the end member components.

unsupervised linear unmixing routine tailored for the OSIRIS data set.

We first filter out all spectra with obvious systematic noise, and those observed at more than $60^{\circ}$ from the sub-solar point. This leaves 1591 high quality spectra from the four observations combined. We bin these to a spectral resolution of $10 \mathrm{~nm}$ for computational feasibility. We input all spectra into $k$-means algorithm (Pedregosa et al. 2011) to find $N$ cluster center spectra. We then find the observed spectra most similar to the $N$ cluster centers, and set these as the initial guesses for candidate end members. This initial degree of similarity is determined by the spectral angle mapping (SAM) metric (Kruse et al. 1993), where the angle $\theta$ between spectra is small for similar spectra and large for dissimilar spectra, and is defined: $\theta=\cos ^{-1}\left(\frac{\boldsymbol{x} \cdot \boldsymbol{y}}{\|\boldsymbol{x}\| \cdot\|\boldsymbol{y}\|}\right)$, where $\boldsymbol{x}$ and $\boldsymbol{y}$ are spectra. We take these $N$ spectra as candidate end members, and model each of the 1591 observed spectra as a linear combination of the $N$ candidate spectra. For each of the 1591 fits, the residual rms is calculated and the rms of each individual fit is summed to give the total rms.

To improve from the initial candidates, we hold all but one of the end member candidates fixed and substitute the remaining candidate with other observed spectra. As before, the substituted spectrum and fixed candidates are used to model all 1591 observed spectra, and the rms of each of the 1591 fits is summed to give the total rms. If the substituted spectrum yields a lower total rms than the previous candidate, it becomes the new candidate. We iterate through all observed spectra to find the best new candidate. The process is repeated successively, alternating which end member is being substituted for. The process ends when the end members have converged, which occurs when a new candidate is not found for $N-1$ successive attempts. This process is illustrated for the $N=3$ case in the ternary plot in Figure 1 . The end member candidates take turns to converge to the best end members at the corners of the plot.

To determine the most appropriate $N$, we use the simple approach of analyzing a range of $N$ and examining the successive residual maps. Once the residual deviation has dropped to the level of noise, spatial structure in the residual maps is lost and the number $N$ of end members used is optimal. We reach this threshold at $N=3$ (Figure 2). For $N=1$ and 2, there is significant spatial structure in the residual maps, indicating compositional regions where the model is inadequate with one or two end members. The rms level drops significantly from $N=1$ (total $\mathrm{rms}=42.7$ ) to $N=2$ (total $\mathrm{rms}=23.6$ ) and from $N=2$ to $N=3$ (total $\mathrm{rms}=11.4$ ), removing nearly all spatial coherence in the residual map. $N=4$ (total $\mathrm{rms}=9.9)$ and $N=5$ (total $\mathrm{rms}=9.4$ ) give only small improvements over $N=3$. Remaining structure in the $N=3$ residuals may indicate further end members, however any further end members would be much less significant, and it is unclear if the remaining structure is related to the physical surface or to systematic noise, so we conservatively choose $N=3$ as the optimal case. As a test of convergence, we repeat the routine for $N=3$ using random initial candidates instead of the cluster center candidates. In 50 tests with random initial guesses, the routine converged to only two results. Both results are qualitatively and quantitatively similar. The better result has slightly lower total rms and was reached for $39(78 \%)$ of the random tests, and is the same result reached when using the initial guess from $k$ means + SAM.

\section{COMPOSITIONAL GEOGRAPHY}

With the approach outlined in the previous section, we find evidence for three distinct compositional units. Their geographic distributions are given by their fractional weights in the linear mixture model (Figure 5). Their orthographic projections at the time of observation are shown in Figure 3, and their cylindrical projections are shown in Figure 4. Component 1 dominates the trailing hemisphere bullseye region, component 2 dominates the leading hemisphere upper latitudes, and component 3 dominates several regions with unique shapes across the leading hemisphere equatorial regions. The most distinct regions of component 3 clearly rotate between the two September 19 observations (Figure 3), further confirming their existence. The end member spectra are located in the middle of the trailing hemisphere $(281 \mathrm{~W}, 4 \mathrm{~S})$, the leading hemisphere northern mid-latitudes $(77 \mathrm{~W}, 32 \mathrm{~N})$, and within Western Powys Regio (155 W, $5 \mathrm{~S}$ ) for components 1, 2, and 3, respectively. Though the end members occupy only one spectral pixel each, linear combinations of these three adequately reproduce all observed spectra across the surface, and yield compositional regions with impressive spatial coherence.

It is interesting that component 3 was not discovered previously in NIMS data. Though more geographically limited, NIMS low resolution data do reach the anti-Jovian regions dominated by component 3 (Carlson et al. 2009). However, previous work investigating compositional distributions at low spatial resolution (McCord et al. 1998, 1999; Carlson et al. 2005; Grundy et al. 2007; Brown \& Hand 2013) focus on ice versus hydrate fractional abundance, and do not attempt to distinguish between different hydrate species. The distinction between different hydrated minerals has been explored in NIMS high resolution data (Dalton et al. 2005, 2012; Dalton 2007; McCord et al. 2010; Shirley et al. 2010), which lacks the necessary surface coverage distinguish global trends. In fact, the anti-Jovian regions dominated by component 3 are distinguishable in NIMS low resolution data, but were not previously recognized as a distinct composition. Western 


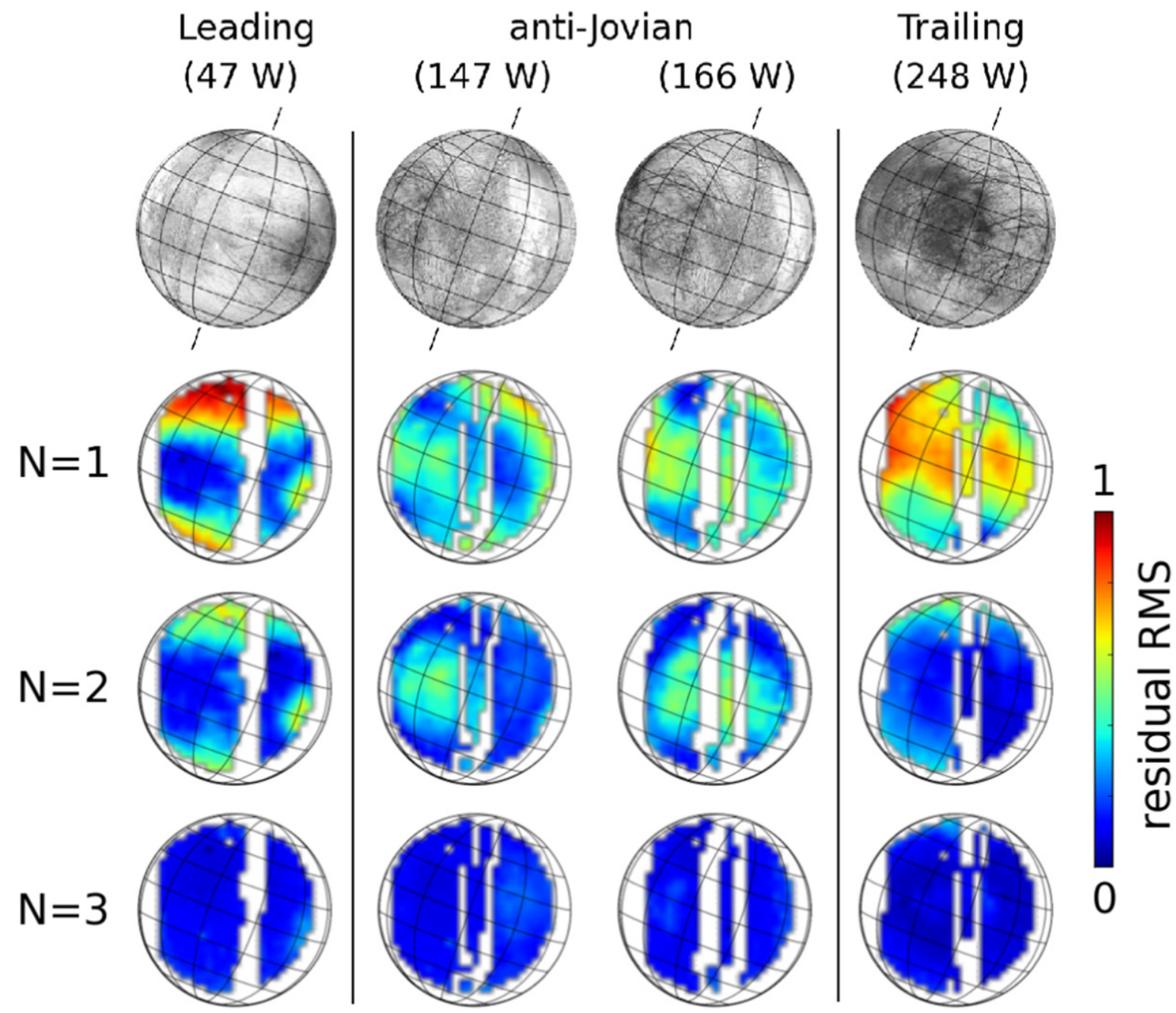

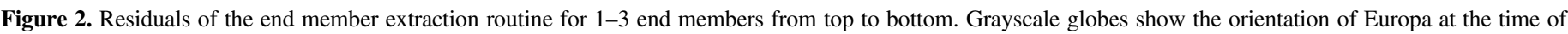

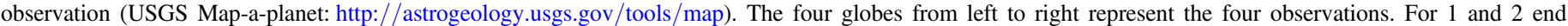

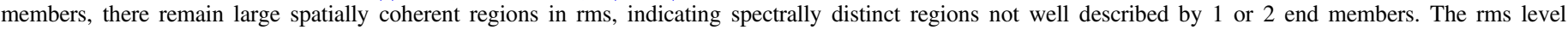

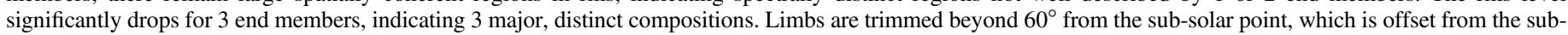
observer point by $8^{\circ} \mathrm{W}$ and $1^{\circ} \mathrm{S}$. Gaps in the data result from stitching separate east/west and $\mathrm{H} / \mathrm{K}$ observations into a complete hyperspectral cube.

Powys Regio particularly stands out in several water-ice abundance maps (McCord et al. 1999; Carlson et al. 2005; Grundy et al. 2007), but was not recognized as distinct from the dominant non-ice component centered on the trailing hemisphere bullseye. Western Powys and the adjacent chaos region at $180 \mathrm{~W}$ longitude are most noticeable in the quality of fit map in plate 2 of McCord et al. (1999), where the poorer quality of fit is clearly associated with these regions, indicating a distinct composition. These regions are also seen in Hansen (2013), which maps the sulfuric acid abundance and average hydrate abundance to NIMS low resolution data; the regions corresponding to component 3 stand out in average hydrate abundance but not in sulfuric acid abundance. Thus NIMS data are consistent with our results.

\section{DISCUSSION: IDENTIFYING COMPOSITION}

The observed spectra of the three compositions are shown in Figure 5. We model each hyperspectral pixel as a linear combination of these three end member spectra, and find that the entire surface at the $\sim 100 \mathrm{~km}$ scale is sufficiently described by a mixture of these three components. Though all three components share the same major spectral features due to water of hydration, they are clearly distinct from each other. Component 1 resembles a darkened and broadened ice spectrum. The features of component 2 are the least distorted and have the strongest band depths, indicating that this component has the greatest fraction of pure water-ice. Component 3 resembles a flattened water-ice spectrum with slightly broadened and shifted features. Distorted water-ice spectra such as this suggest hydrated species (McCord et al. 1998, 1999, 2002), though the lack of additional features makes a unique identification difficult.

The geographic distribution of each component is a strong indication of its identity. The distribution of component 1 is globally symmetric, centered on the trailing hemisphere equator. This is consistent with the known patterns of enhanced irradiation and bombardment from the Jovian magnetosphere (Carlson et al. 1999b, 2002; Paranicas et al. 2001; Cassidy et al. 2013; Dalton et al. 2013a). This strongly suggests that component 1 is exogenous, or initially endogenous but heavily altered by exogenous processes. Component 2 dominates the leading hemisphere polar regions, consistent with the known distribution of pure water ice or deposited water ice frost 
Leading $(47 \mathrm{~W})$
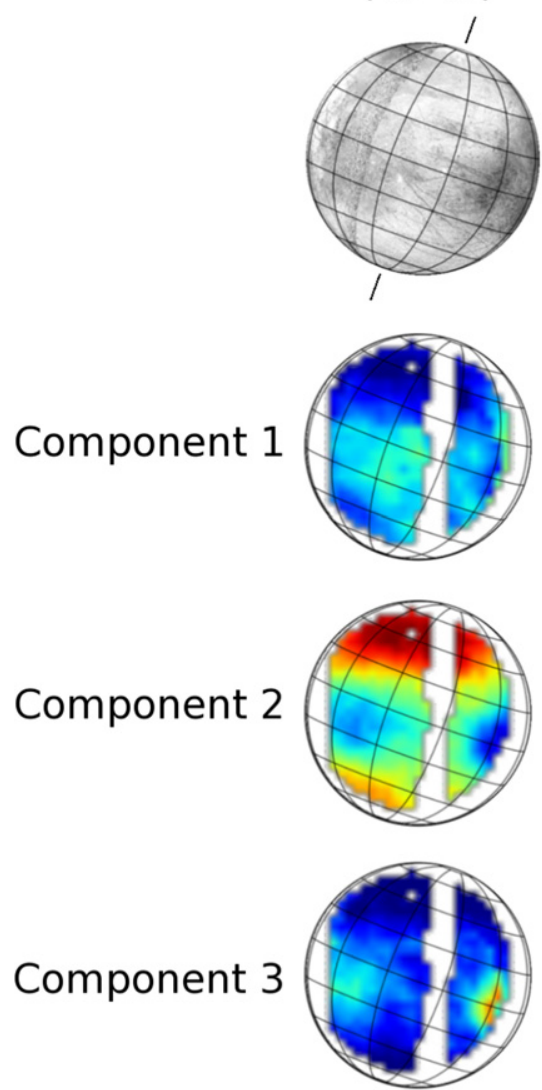

anti-Jovian

(147 W)

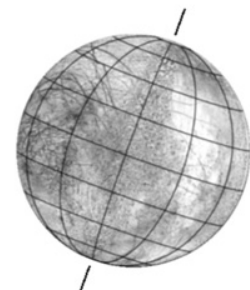

I
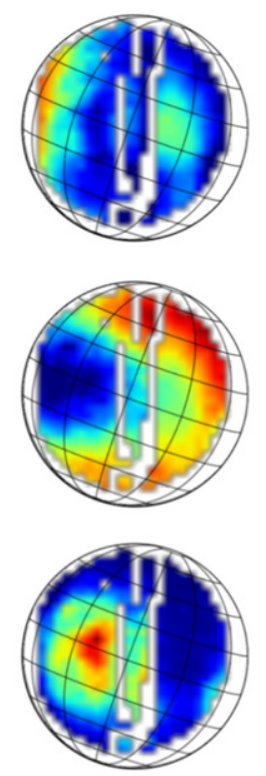

$(166 \mathrm{~W})$
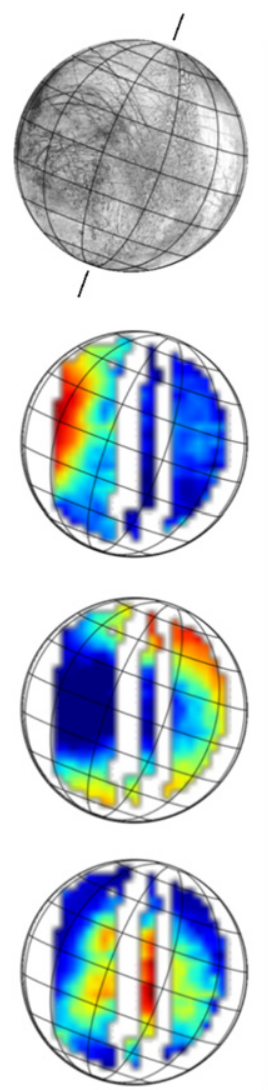

Trailing

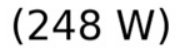

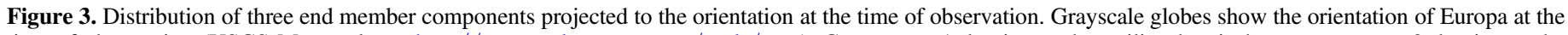

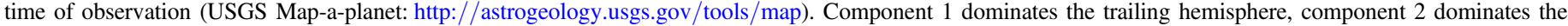

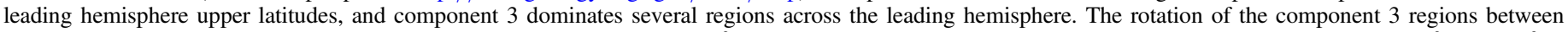

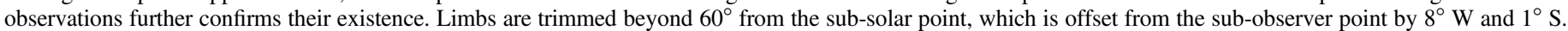
Gaps in the data result from stitching separate east/west and $\mathrm{H} / \mathrm{K}$ observations into a complete hyperspectral cube.

(Grundy et al. 2007; Brown \& Hand 2013). Unlike components 1 and 2 , the distribution of component 3 is not globally symmetric, nor is it consistent with regions known to be compositionally distinct. The shapes of the regions dominated by component 3 are revealing; they are markedly similar to geologic units of chaos as mapped by Doggett et al. (2009), to within the angular resolution of our observations. Though the spectral pixel identified as component 3 is located within Western Powys Regio, it also dominates the abundance in other regions; Tara Regio chaos in particular is reproduced remarkably well (Figures 3 and $4, \sim 85 \mathrm{~W}$ ). Chaos regions are believed to represent areas of recent interaction with the subsurface, and a variety of mechanisms have been proposed including widescale diapiric upwelling (Pappalardo et al. 1998), melt-through (Greenberg et al. 1999), and lens-collapse (Schmidt et al. 2011). Thus, the association of component 3 with chaos suggests an initially endogenous composition, less altered by exogenous processes than the trailing hemisphere. The trailing hemisphere is also covered with large chaos regions, in which we see no evidence for component 3 . Our interpretation is that component 3 contains the greatest fraction of salts derived from the subsurface, possibly a brine or evaporite deposit. The lack of component 3 on the trailing hemisphere is due to enhanced exogenous alteration, where the surface has been coated with a layer of irradiation products, primarily sulfuric acid hydrate (Carlson et al. 1999b). An endogenous composition likely exists at greater depth in trailing hemisphere chaos regions, where it is shielded from exogenous processes and NIR remote sensing.

It was suggested from NIMS spectra that hydrated salts are abundant on Europa's surface, particularly $\mathrm{Na}$ - and Mg-sulfates (McCord et al. 1998). Laboratory spectra of these salts have been used extensively to model high spatial resolution NIMS data (Dalton et al. 2005, 2012; Dalton 2007; Shirley et al. 2010). Following these analyses, we perform similar linear mixture modeling to the OSIRIS data. The results are shown in Figure 6. In the fits shown, we include $\mathrm{H}_{2} \mathrm{SO}_{4}$ hydrate, mirabilite $\left(\mathrm{Na}_{2} \mathrm{SO}_{4} \cdot 10 \mathrm{H}_{2} \mathrm{O}\right)$, hexahydrite $\left(\mathrm{MgSO}_{4} \cdot 6 \mathrm{H}_{2} \mathrm{O}\right)$, bloedite $\left(\mathrm{Na}_{2} \mathrm{Mg}\left(\mathrm{SO}_{4}\right)_{2} \cdot 4 \mathrm{H}_{2} \mathrm{O}\right), \mathrm{MgSO}_{4}$ brine, and water ice at grain sizes of $50,75,100,250$, and $1000 \mu \mathrm{m}$. The fit to component 1 is generally satisfying. This method finds that component 1 is dominated by $\mathrm{H}_{2} \mathrm{SO}_{4}$ with lesser amounts of the modeled salts and water ice, consistent with an exogenous interpretation. Furthermore, this method finds that component 3 is slightly dominated by hydrated salts, with a significant but lesser amount of fine-grained water ice and little $\mathrm{H}_{2} \mathrm{SO}_{4}$ hydrate, consistent with an endogenous interpretation for component 3 .

Though this result is enticing, the quality of the spectral fit is unacceptably poor (Figure 6). Although a linear mixture of 

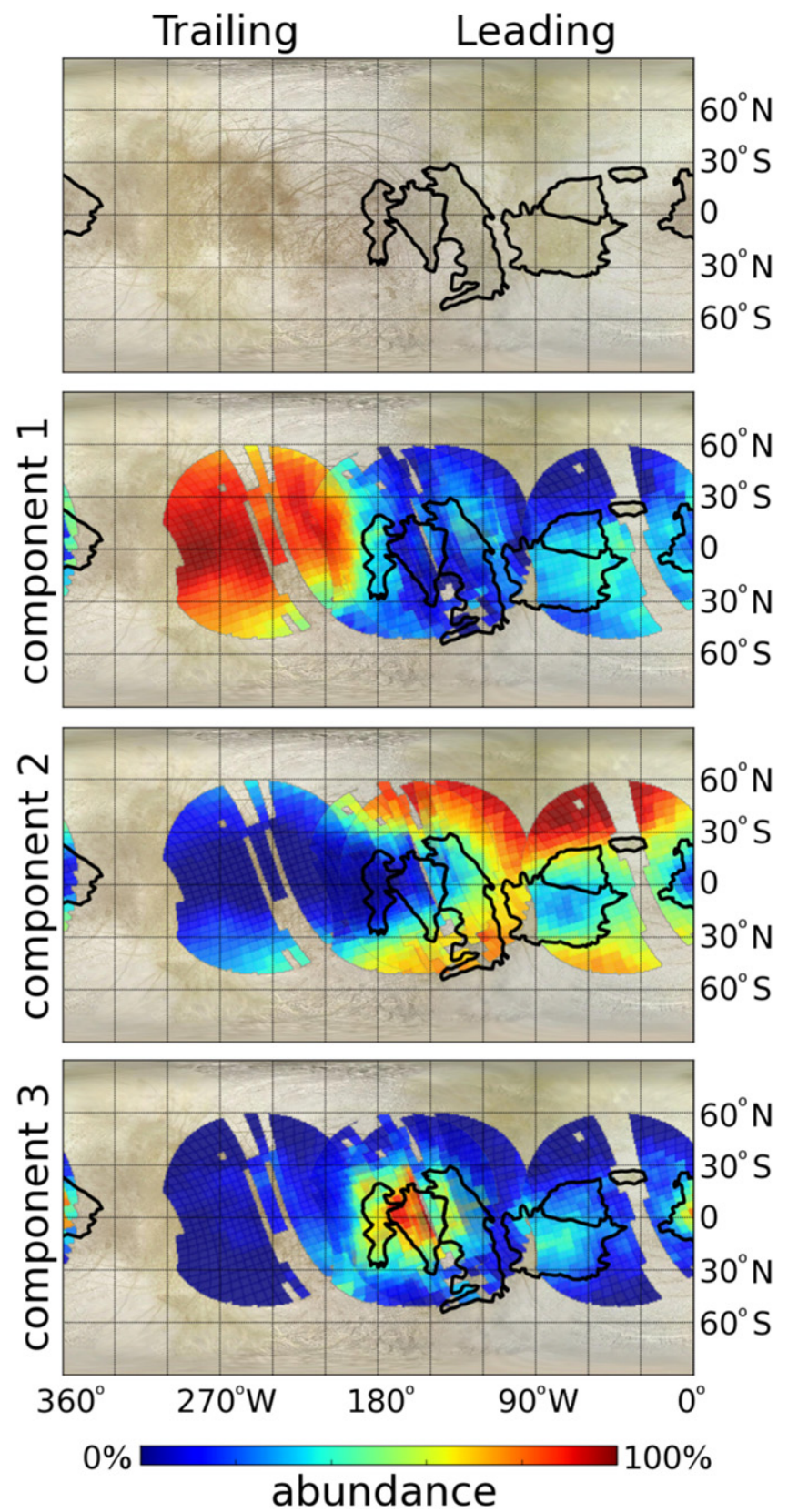

Figure 4. Distribution of components 1-3 mapped to a cylindrical projection Overplotted in black are approximate locations of large leading hemisphere chaos regions from Doggett et al. (2009). Component 1 dominates the trailing hemisphere bullseye and component 2 dominates the leading hemisphere upper latitudes, in good agreement with known "non-icy" and "icy" Europa. Component 3 is associated with leading hemisphere chaos regions to within the angular resolution of the observations, suggesting an endogenous origin.

these species is able to match the coarse continuum level, it is not able to fit the shape and depth of distinct features in the $\mathrm{H}$ band, or the distorted shape of the $2.2 \mu \mathrm{m}$ peak in the $\mathrm{K}$ band. This remains true even when we relax the constraints of the linear mixture model: in the fits shown, we include a dark neutral substance with a spectral slope, and do not restrict the sum of the components to match $100 \%$. Figure 7 compares the observed spectra to the laboratory spectra. Conversely, Figure 7 shows significant differences between component 3 and the hydrated sulfate salts considered. Each of these salts possesses distinct features that should be seen in the observed spectra, if

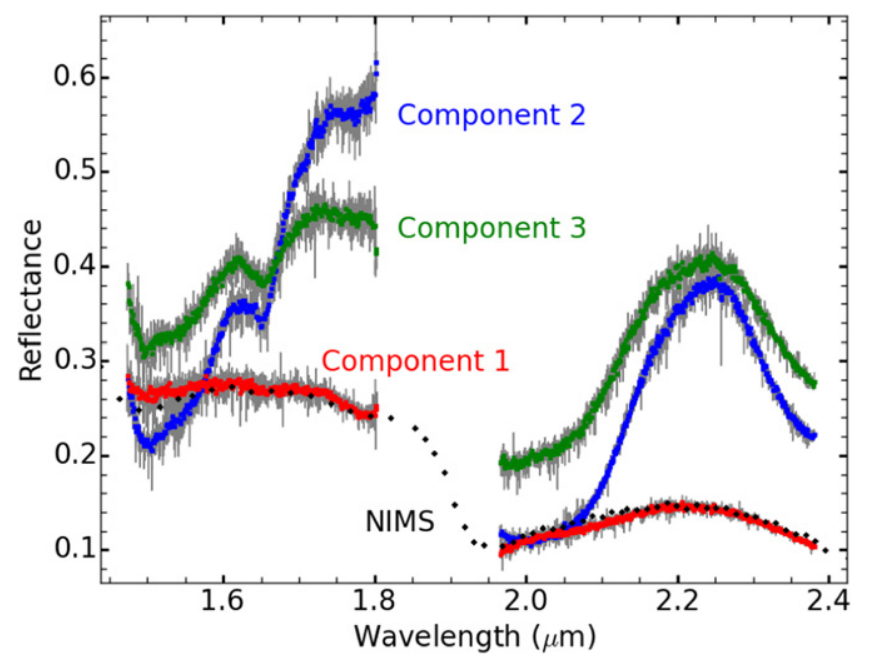

Figure 5. OSIRIS spectra of Europa's three dominant end members at the $\sim 100 \mathrm{~km}$ scale. Gray lines show the true spectral resolution; colored spectra are binned to a resolution of $1 \mathrm{~nm}$. The NIMS Europa hydrate spectrum (Carlson et al. 1999b) is overplotted in black and scaled for comparison. Note the similarity of NIMS to component 1, though at a much coarser resolution.

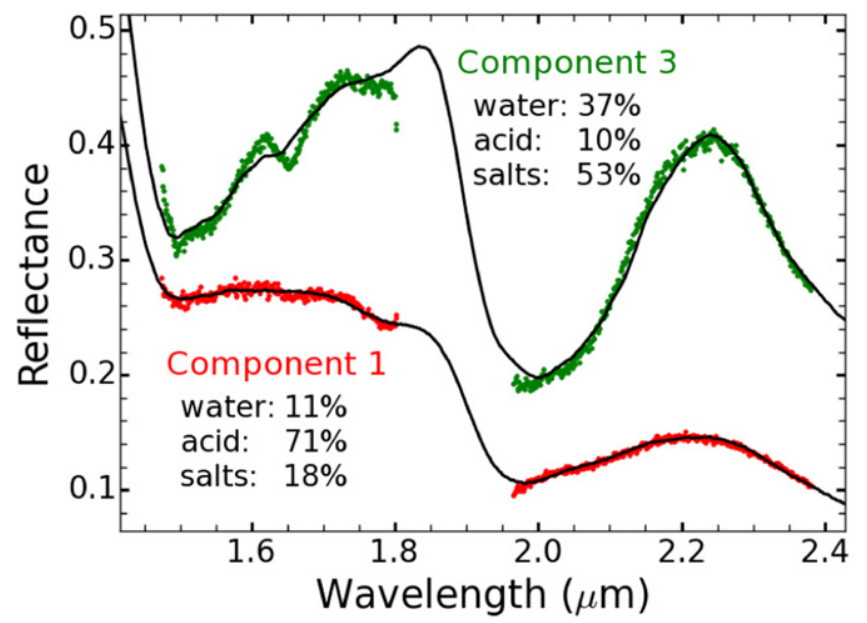

Figure 6. Linear mixture fits to the non-ice components, following the method of Dalton (2007). At moderate spectral resolution, this library describes component 1 well and component 3 poorly. The result for component 1 is $71 \%$ $\mathrm{H}_{2} \mathrm{SO}_{4} \cdot n \mathrm{H}_{2} \mathrm{O}, 11 \%$ bloedite, $7 \% 250 \mu \mathrm{m} \mathrm{H}_{2} \mathrm{O}$ ice, $4 \%$ mirabilite, $4 \% 1000 \mu \mathrm{m}$ $\mathrm{H}_{2} \mathrm{O}$ ice, $3 \%$ hexahydrite, and $<1 \% \mathrm{MgSO}_{4}$ brine and $\mathrm{H}_{2} \mathrm{O}$ ice at 50, 75, $100 \mu \mathrm{m}$. The result for component 3 is $42 \%$ bloedite, $37 \% 50 \mu \mathrm{m} \mathrm{H}_{2} \mathrm{O}$ ice, $11 \%$ mirabilite, $10 \% \mathrm{H}_{2} \mathrm{SO}_{4} \cdot n \mathrm{H}_{2} \mathrm{O}$, and $<1 \% \mathrm{MgSO}_{4}$ brine, hexahydrite, and $\mathrm{H}_{2} \mathrm{O}$ ice at $75,100,250$, and $1000 \mu \mathrm{m}$.

they were present in abundance. Though the results of linear mixtures with these salts are consistent with an endogenous interpretation, the individual sulfate salts considered cannot be present in abundance.

At the observed wavelengths, the defining spectral characteristics of component 3 are the distortion of water-ofhydration features and the lack of additional, non-water-ice features. Thus the species making up component 3 must share these characteristics. One possible alternative composition is anhydrous chloride salts such as $\mathrm{NaCl}, \mathrm{KCl}$ (Figure 8). Note that the term anhydrous is relative, e.g., baking at $400^{\circ} \mathrm{C}$ for days is not sufficient to remove all of the bound water, so residual features persist (Hanley et al. 2014). In the case of $\mathrm{NaCl}$, the anhydrous phase is more spectrally similar to the observed spectra than the brine phase (Figures 1 and 4 in 


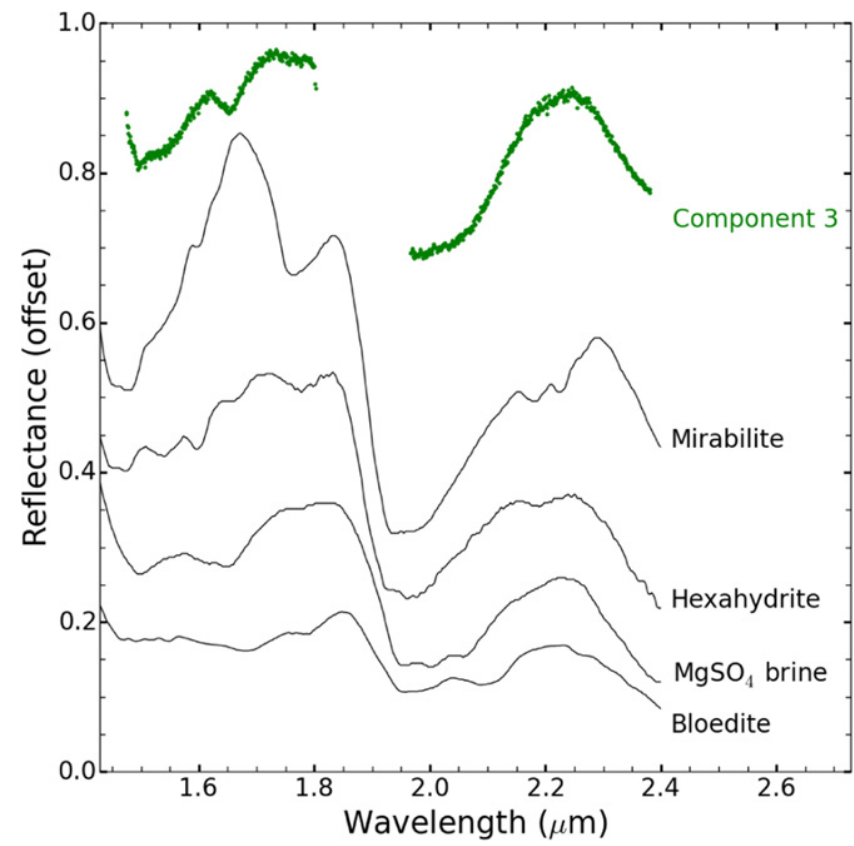

Figure 7. Spectrum of component 3 compared to spectra of various hydrated sulfate salts (Dalton et al. 2005) traditionally thought to be abundant on Europa's surface. Note their spectral dissimilarity to the OSIRIS spectrum of component 3, representing potentially the saltiest composition on Europa. At sufficiently fine spectral resolution, hydrated sulfate minerals possess many distinct features not seen in the observed spectra, constraining their surface abundances. Spectra are offset for clarity by $0.5,0.13,0.07,-0.18$, and 0.0 from top to bottom.

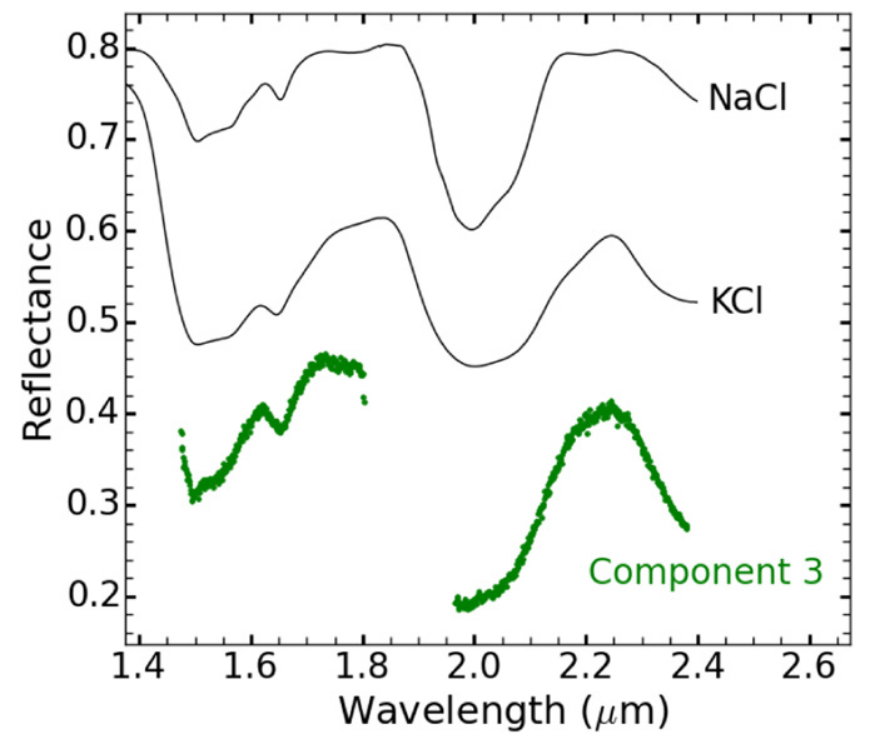

Figure 8. Spectrum of component 3 compared to alternative salt compositions Anhydrous $\mathrm{NaCl}$ and $\mathrm{KCl}$ (Brown \& Hand 2013) are similarly smooth. Importantly, they lack the additional features characteristic of hydrated sulfate minerals that are not seen in the observed spectra.

Hanley et al. 2014). However, the chlorate and perchlorate salts from Hanley et al. (2014) possess distinct spectral features not seen in the observed spectra. Anhydrous salts could indeed be present on Europa's surface; evaporite deposits are speculated to result from exposure of a brine to Europa's surface conditions through the processes of sublimation, freezing, and flash evaporation (McCord et al. 1998). A search through the literature of published spectra at conditions relevant to
Europa and spectral resolution comparable to OSIRIS revealed $\mathrm{NaClO}$ and $\mathrm{NaOH}$ (Brown \& Hand 2013), which are conceivable radiolytic products of a $\mathrm{NaCl}-\mathrm{H}_{2} \mathrm{O}$ substrate, and $\mathrm{NaHCO}_{3}$ brine (Dalton et al. 2005), which is a plausible endogenous species. Other carbonate salts are also plausible, but must be investigated at appropriate conditions and moderate spectral resolution.

\section{DISCUSSION: REVISITING THE SULFATE HYPOTHESIS}

The traditional view that Europa's endogenous non-ice component is dominated by $\mathrm{Mg}$-sulfates traces back at least as far as Kargel (1991), which found the system $\mathrm{MgSO}_{4}-$ $\mathrm{Na}_{2} \mathrm{SO}_{4}-\mathrm{H}_{2} \mathrm{O}$ to be the favored product of aqueously differentiated carbonaceous chondrites in conditions expected for icy satellites. This served as a working hypothesis for early NIMS investigations such as McCord et al. (1998), which found an expected spectral agreement between NIMS and sulfate salts. This story was further supported by chondriteleaching experiments (Fanale et al. 2001) and subsequent geochemical modeling (Zolotov \& Shock 2001). Later NIMS investigations showed that the spectra are better explained by mixtures of sulfuric acid and hydrated salts, but still focus solely on sulfate salts (Dalton 2007; Shirley et al. 2010; Dalton et al. 2012, 2013b). These results made for a satisfying, consistent story. However, there are many caveats to this story, and a growing body of work is challenging the traditional view.

The spectroscopic argument set in motion by early NIMS investigations relied on an even smaller spectral library than exists today, among which hydrated sulfate salt minerals were one of the few items. Furthermore, NIMS investigations face the challenge of low spectral resolution; the spectral similarity among hydrated species makes the ability to spectrally distinguish between them difficult, and this difficulty is exacerbated at low spectral resolution, where small features are smoothed out and the subtle but significant shape of the continuum is less apparent. OSIRIS observations show that the NIR moderate resolution spectra of the entire surface resembles water-ice, precluding the possibility for species with distinct non-ice spectral features. The only exception to this is the $2.07 \mu \mathrm{m}$ feature attributed to epsomite (Brown \& Hand 2013). Ironically, the identification of epsomite challenges the case for endogenous sulfates, due to the geographical association of epsomite with exogenous and not endogenous processes. The OSIRIS spectra are in good agreement with similar observations from Spencer et al. (2006), which resolve ice and non-ice units and find equally smooth, featureless spectra. Spencer et al. (2006) also conclude that higher resolution spectra are inconsistent with the crystalline hydrates considered.

Geochemical models predicting surface composition also claim a large degree of uncertainty. Kargel (1991) notes that sufficiently alkaline conditions during aqueous alteration would stabilize $\mathrm{Mg}$ and $\mathrm{Fe}$ in $\mathrm{Mg} / \mathrm{Fe}$-hydroxides, preventing significant amounts of $\mathrm{Mg}$ in the solution, or that sufficiently acidic conditions could favor more abundant carbonates in solution. Kargel et al. (2000) explores a wide variety of alternative surface compositions, and for example notes that the hydrothermal processes causing a $\mathrm{NaCl}$ ocean on Earth could also occur on Europa under the right conditions. McKinnon \& Zolensky (2003) raise many objections to the models favoring a $\mathrm{MgSO}_{4}$-dominated system, including a possibly incorrect initial composition, the irrelevant conditions of chondrite 
leaching experiments of Fanale et al. (2001), and the difficulty of forming sulfate in reducing conditions. However, though surface and ocean composition are certainly related, the exact relationship is uncertain; an alternative surface composition suggests but does not necessarily require an alternative ocean composition. For example, Zolotov \& Shock (2001) point out that freezing leads to brines of different composition than the ocean, and that endogenous surface salts may only be the end member of a compositional stratification throughout the ice shell.

Europa's known atmospheric composition further challenges the traditional view. The tenuous atmosphere is produced by sputtering of surface material (Johnson et al. 1998); thus surface and atmosphere compostion are intimately related. The presence of $\mathrm{Na}$ (Brown \& Hill 1996) and K (Brown 2001) in the sputtered atmosphere is established, and their relative abundances provide a compelling argument for an endogenous source (Brown 2001). Furthermore, the non-detection of atmospheric Mg (Horst \& Brown 2013) constrains its surface abundance and challenges the traditional view of an endogenous surface component dominated by the $\mathrm{Mg}$ cation.

Enceladus may represent an analogous environment to Europa, and Enceladus' plume composition also suggests an alternative salty component. $\mathrm{Na}, \mathrm{K}, \mathrm{Cl}$, and $\mathrm{CO}_{3}$ have all been detected, whereas $\mathrm{Mg}$ and $\mathrm{SO}_{4}$ have not (Postberg et al. 2009, 2011). Using a geochemical model based on the empirical evidence, Glein et al. (2015) favor an Enceladus ocean composition of $\mathrm{Na}-\mathrm{Cl}-\mathrm{CO}_{3}$.

Furthermore, recent work by Hand \& Carlson (2015) shows that irradiated alkali halides such as $\mathrm{NaCl}$ can describe the yellow to brown colors and coarse continuum level of Europa's visible spectrum. The formation of similar color centers in sulfate salts is less certain but many sulfates bleach to white rapidly after irradiation or stay colorless in the visible even after irradiation (Nash \& Fanale 1977, K. P. Hand et al. 2015, in preparation). Previously, the darker colors seen by Galileo Solid State Imager were attributed to a mixture with an unknown associated species that is featureless in the infrared (Dalton et al. 2005; McCord et al. 2010).

This work further supports that the traditional view of Europa's endogenous surface composition must be reconsidered. We find evidence that Europa's surface consists of three distinct compositional units at the $\sim 100 \mathrm{~km}$ scale. We identify the endogenous composition based on association with geologic chaos units, and show that its spectrum is inconsistent with hydrated sulfate minerals at moderate resolution.

The implications of alternative salts for habitability are intriguing but difficult to interpret, even with a complete knowledge of surface composition and subsurface exchange. We can speculate that the dominant ocean salt has implications for salinity, which may be a limiting factor for the origin of life and the ability for life to thrive. Though the salinity of the Europan ocean is only marginally constrained, an ocean dominated by $\mathrm{Na}^{+}$and $\mathrm{Cl}^{-}$could imply a lower salinity compared to $\mathrm{Mg}^{2+}$ and $\mathrm{SO}_{4}^{2-}$, by a factor of $\sim 3$ for the upper limit of measured ocean conductivity (Hand \& Chyba 2007). We can also speculate that biological processes, if present, may manifest themselves in the endogenous surface composition. For example, a Europan biosphere supported by sulfate reduction (Zolotov \& Shock 2003) could result in an ocean depleted in sulfate, if the supply of sulfate is the limiting factor for biomass production. This scenario is likely inconsistent with a surface abundant in endogenous sulfate. Similarly, a Europan biosphere supported by methanogenesis could yield a relatively reduced methane-rich ocean or a relatively oxidized sulfate- and bicarbonate-rich ocean (McCollom 1999), leading to an endogenous surface enhaced in the respective species. However, abioligical processes are likely able to explain surface composition as well, and such interpretations should be favored while definitive evidence for biological activity is lacking.

\section{CONCLUSIONS}

We find evidence for three distinct compositions across Europa's surface at the $\sim 100 \mathrm{~km}$ scale, from hyperspectral observations with Keck II OSIRIS and adaptive optics. The first component dominates the trailing hemisphere bullseye and the second component dominates the leading hemisphere upper latitudes, consistent with regions previously found to be dominated by irradiation products and water ice, respectively. The third component is geographically associated with large geologic units of chaos, suggesting an endogenous identity. This is the first time that the endogenous hydrate species has been mapped at a global scale.

Europa's moderate resolution surface spectra in the NIR are ubiquitously smooth, distorted water-ice spectra. The spectrum of component 3 is not consistent with linear mixtures of the current spectral library. In particular, the hydrated sulfate minerals previously favored possess distinct spectral features that are not present in the spectrum of component 3 , and thus cannot be abundant at large scale. One alternative composition is chloride evaporite deposits, possibly indicating an ocean solute composition dominated by the $\mathrm{Na}^{+}$and $\mathrm{Cl}^{-}$ions.

The traditional view that Europa's non-ice endogenous surface is dominated by hydrated $\mathrm{Mg}$-sulfates must be reconsidered. Higher resolution surface spectra at greater geographic coverage, as well as insight from new laboratory irradiation experiments, atmospheric composition, and analogy to Enceladus all point toward a new understanding of Europa's endogenous hydrate.

This research was supported by grant 1313461 from the National Science Foundation. K. P. H. acknowledges support from the Jet Propulsion Laboratory, California Institute of Technology, under a contract with the National Aeronautics and Space Administration and funded in part through the internal Research and Technology Development program. The data presented herein were obtained at the W. M. Keck Observatory, which is operated as a scientific partnership among the California Institute of Technology, the University of California, and the National Aeronautics and Space Administration. The Observatory was made possible by the generous financial support of the W. M. Keck Foundation. The authors wish to recognize and acknowledge the very significant cultural role and reverence that the summit of Mauna Kea has always had within the indigenous Hawaiian community. We are most fortunate to have the opportunity to conduct observations from this mountain.

\section{REFERENCES}

Brown, M. E. 2001, Icar, 151, 190

Brown, M. E., \& Hand, K. P. 2013, AJ, 145, 110

Brown, M. E., \& Hill, R. E. 1996, Natur, 380, 229

Carlson, R. W., Anderson, M. S., Johnson, R. E., et al. 1999a, Sci, 283, 2062 
Carlson, R. W., Anderson, M. S., Johnson, R. E., Schulman, M. B., \& Yavrouian, A. H. 2002, Icar, 157, 456

Carlson, R. W., Anderson, M. S., Mehlman, R., \& Johnson, R. E. 2005, Icar, 177,461

Carlson, R. W., Calvin, W. M., Dalton, J. B., et al. 2009, in Europa, ed. R. T. Pappalardo et al. (Tucson, AZ: Univ. Arizona Press), 283

Carlson, R. W., Johnson, R. E., \& Anderson, M. S. 1999b, Sci, 286, 97

Cassidy, T., Paranicas, C. P., Shirley, J. H., et al. 2013, P\&SS, 77, 64

Chyba, C. F. 2000, Natur, 403, 381

Dalton, J. B. 2007, GeoRL, 34, L21205

Dalton, J. B., Cassidy, T., Paranicas, C., et al. 2013a, P\&SS, 77, 45

Dalton, J. B., Prieto-Ballesteros, O., Kargel, J. S., et al. 2005, Icar, 177, 472

Dalton, J. B., Shirley, J. H., \& Kamp, L. W. 2012, JGRE, 117, E03003

Dalton, J. B., Shirley, J. H., Prockter, L. M., et al. 2013b, LPSC, 44, 3011

Doggett, T., Greeley, R., \& Figueredo, P. H. 2009, in Europa, ed. R. T. Pappalardo et al. (Tucson, AZ: Univ. Arizona Press), 137

Fanale, F. P., Li, Y.-H., De Carlo, E., et al. 2001, JGRE, 106, 14595

Glein, C. R., Baross, J. A., \& Waite, J. H., Jr. 2015, GeCoA, 162, 202

Greenberg, R., Hoppa, G. V., Tufts, B. R., Geissler, P., \& Riley, J. 1999, Icar, 141,263

Grundy, W. M., Burratti, B. J., Cheng, A. F., et al. 2007, Sci, 318, 234

Hand, K. P., \& Carlson, R. W. 2015, GeoRL, 42, 3174

Hand, K. P., \& Chyba, C. F. 2007, Icar, 189, 424

Hand, K. P., Chyba, C. F., Priscu, J. C., et al. 2009, in Europa, ed. R. T. Pappalardo et al. (Tucson, AZ: Univ. Arizona Press), 283

Hanley, J., Dalton, J. B., Chevrier, V. F., Jamieson, C. S., \& Barrows, R. S. 2014, JGRE, 119, 2370

Hansen, G. B. 2013, LPSC, 44, 2998

Horst, S. M., \& Brown, M. E. 2013, ApJL, 764, L28

Johnson, R. E., Killen, R. M., Waite, J. H., Jr., \& Lewis, W. S. 1998, GeoRL, 25, 3257

Johnson, R. E., Lanzerotti, L. J., \& Brown, W. L. 1982, NucIM, 198, 147

Kargel, J. S. 1991, Icar, 94, 368
Kargel, J. S., Kaye, J. Z., Head, J. W., et al. 2000, Icar, 148, 226

Kattenhorn, S. A., \& Prockter, L. M. 2014, NatGe, 7, 762

Kivelson, M. G., Khurana, K. K., Russell, T. T., et al. 2000, Sci, 289, 1340

Kruse, F. A., Lefkoff, A. B., Boardman, J. W., et al. 1993, RSEnv, 44, 145

Kuiper, G. P. 1957, AJ, 62, 245

Lane, A. L., Nelson, R. M., \& Matson, D. L. 1981, Natur, 292, 38

Lanzerotti, L. J., Brown, W. L., Poate, J. M., \& Augustyniak, W. M. 1978, GeoRL, 5, 155

Larkin, J. E., Quirrenbach, A., Krabbe, A., et al. 2003, Proc. SPIE, 4841, 1600

McCollom, T. M. 1999, JGRE, 104, 30729

McCord, T. B., Hansen, G. B., Combe, J.-P., \& Hayne, P. 2010, Icar, 209, 639

McCord, T. B., Hansen, G. B., Fanale, F. P., et al. 1998, Sci, 280, 1242

McCord, T. B., Hansen, G. B., Matson, D. L., et al. 1999, JGRE, 104, 3311

McCord, T. B., Teeter, G., Hansen, G. B., et al. 2002, JGRE, 107, 5004

McKinnon, W. B., \& Zolensky, M. E. 2003, AsBio, 3, 879

Nash, D. B., \& Fanale, F. P. 1977, Icar, 31, 40

Pappalardo, R. T., \& Barr, A. C. 2004, GeoRL, 31, L01701

Pappalardo, R. T., Head, J. W., Greeley, R., et al. 1998, Natur, 391, 365

Paranicas, C., Carlson, R. W., \& Johnson, R. E. 2001, GeoRL, 28, 673

Peddinti, D. A., \& McNamara, A. K. 2015, GeoRL, 42, L063950

Pedregosa, F., Varoquaux, G., Gramfort, A., et al. 2011, Journal of Machine Learning Research, 12, 2825

Postberg, F., Kempf, S., Schmidt, J., et al. 2009, Natur, 459, 1098

Postberg, F., Schmitt, J., Hillier, J., Kempf, S., \& Srama, R. 2011, Natur, 474,620

Schmidt, B. E., Blankenship, D. D., Patterson, G. W., \& Schenk, P. M. 2011, Natur, 479, 502

Shirley, J. H., Dalton, J. B., Prockter, L. M., \& Kamp, L. W. 2010, Icar, 210,358

Spencer, J. R., Grundy, W. M., Dumas, C., et al. 2006, Icar, 182, 202

Zolotov, M. Y., \& Shock, E. L. 2001, JGRE, 106, 32815

Zolotov, M. Y., \& Shock, E. L. 2003, JGRE, 108, 5022 